\title{
Strontium ranelate - a novel drug for osteoporosis with a dual mechanism of action
}

\author{
Helen Barham and Janet Marianne Jackson \\ CSF Medical Communications, Cheltenham, UK \\ CSF Medical Communications, Auckland, New Zealand
}

Drugs in Context

DOI: http://dx.doi.org/10.7573/dic.212200

Citation: Barham H, Jackson JM. Strontium ranelate - a novel drug for osteoporosis with a dual mechanism of action. Drugs in Context: e212200. doi: $10.7573 /$ dic. 212200 .

Copyright: this is an open access article published under the terms of the Creative Commons License Deed (CG BY-NG-ND 3.0) which allows you to share, copy, distribute and transmit the work provided it is properly attributed. You may not use this work for commercial purposes. For further information on commercial use, contact publisher@justmedicalmedia.com or go to wrerw.drugsincontext.com/copyright.

Search criteria: English language articles were identified by searching the PubMed database using the search terms 'strontium ranelate' and 'Protelos'. Abstracts were evaluated and selected for further review according to our standard protocols. Bibliographies of individual articles were also assessed for additional articles of interest and the manufacturer of strontium ranelate was contacted and was invited to supply any additional data to that identified via the PubMed database.

Date of last literature search: 1 November 2008.

Conclusion: Strontium ranelate promotes bone formation and reduces bone resorption via an innovative mechanism of action. It is the only drug for which a reduction in fracture risk sustained over 8 years' treatment has been demonstrated. It has a favourable side-effect profile, a convenient dosing regime and has been shown to be of particular benefit in the elderly.

NB: This article was originally published by CSF Medical Communications Ltd (CSF) in Drugs in Context 2008;4:239-54. Drugs in Context and all CSF copyrights were acquired by Just Medical Media Ltd in 2009. 


\section{SUMMARY}

Osteoporotic fractures are a major cause of morbidity and mortality and exert a huge economic and social burden - but one that is largely preventable by the identification of at-risk patients and the use of antiosteoporotic drugs developed over the last two decades, the most recent and innovative of which is strontium ranelate (SR). SR is a dual agent that reduces bone resorption and simultaneously increases bone formation. It increases bone mineral density (BMD) at the spine and hip in postmenopausal women with osteoporosis, and can normalise BMD in women with osteopenia. More importantly, it is the only drug for which a reduction in fracture risk sustained over 8 years' treatment has been demonstrated. Whilst all the anti-osteoporotic medications currently available reduce vertebral fractures, only SR and the bisphosphonates alendronate and risedronate have been shown to reduce hip fractures. This is particularly important given the high mortality and morbidity associated with hip fracture. Furthermore, SR is the only anti-osteoporotic medication that has been shown to reduce fracture risk in the very elderly ( $\geq 80$ years). This group of patients are particularly vulnerable because of their decreased bone strength and increased propensity to falls. SR is convenient to take once daily as granules dissolved in water, preferably at bedtime. It is generally well tolerated, is not associated with gastritis, and does not interact with commonly used drugs, other than calcium-containing preparations and foods, and some antibiotics. Recent analyses have shown that SR is cost-effective in the treatment of osteoporosis. Thus, SR is a useful addition to the range of anti-osteoporotic medications available, offering advantages over the bisphosphonates by virtue of its better tolerability, especially in terms of gastritis, and easier dosage, and offers significant benefits for the very elderly.

Key words: strontium ranelate; Protelos ${ }^{\circledR}$; osteoporosis; bone resorption

\section{OSTEOPOROSIS: A PERSPECTIVE}

The lifetime risk for wrist, hip and vertebral fracture - the characteristic sites of osteoporotic fractures - has been estimated at $15 \%$, which is similar to that for coronary heart disease. ${ }^{1}$

In Europe there were 3.79 million osteoporotic fractures in 2000 , costing $€ 31.7$ billion. ${ }^{2}$ In the UK, osteoporosis results in over 310,000 fractures annually, including 70,000 hip fractures, 70,000 wrist fractures and 120,000 vertebral fractures. ${ }^{3}$ The incidence of hip fracture is rising by $1-3 \%$ per year in most parts of the world ${ }^{4}$ and is expected to increase from 1.66 million worldwide (three-quarters of these in women) in 1990 to more than 6 million cases per year by $2050 .{ }^{5}$ The incidence of hip fracture in women is twice that in men in the UK and USA. ${ }^{4}$

Fractures can cause severe pain, disability, restricted mobility and premature death. ${ }^{4}$ Although only about one-third of vertebral fractures are clinically diagnosed ${ }^{6}$ these fragility fractures can cause outward curvature of the thoracic spine, height loss, unexplained chronic pain and functional difficulties, all of which can compromise quality of life. In a prospective study of 667 men and women with osteoporosis, mortality was 4.4-fold higher in patients with vertebral fractures than in those without. ${ }^{7}$

Hip fractures are the most devastating fracture; $10-20 \%$ of women die earlier than expected for their age within the first year of sustaining a fracture, and at 1 year hip fracture is associated with $20 \%$ mortality, whilst $50 \%$ of patients are unable to live independently. ${ }^{6}$

The World Health Organization has estimated that, worldwide, osteoporosis affects $5 \%$ of women and $2.4 \%$ of men under 50 years of age, rising to $50 \%$ of women and $20 \%$ of men over 85 years of age. ${ }^{6}$ In Europe, Japan and the USA, more than 75 million people are affected by the condition. ${ }^{1}$ The prevalence is increasing with the aging of the general population. ${ }^{6}$

The treatment of osteoporotic fractures represents a huge - but preventable - economic 
burden. In the UK, the annual cost of providing social care and support for patients sustaining fractures is estimated at $£ 1.8$ billion. ${ }^{8}$ The hospitalisation and treatment of hip fractures - which invariably require surgical repair or replacement of the joint - costs the UK NHS over £940 million annually. ${ }^{9}$ Recent cost projections suggest that osteoporotic fractures in women will cost $£ 2.1$ billion (€3.04 billion) by $2010 .{ }^{10}$ Given that $20-30 \%$ of symptomatic hip and spine fractures occur in men in affluent countries a further $£ 0.5$ billion can be added to this. ${ }^{11}$

\section{The importance of diagnosis}

Osteoporosis is defined by the WHO as BMD at least 2.5 SD below the mean BMD of the young adult (T-score $\leq-2.5) \cdot{ }^{6}$ These criteria were specifically developed for the spine and/or hip in postmenopausal women but are also likely to apply to men. ${ }^{12}$ The predictive validity of BMD for bone fracture is considered to be at least as accurate as that of blood pressure for stroke. ${ }^{13}$

Given that osteoporosis is largely 'silent', in the past the condition was not diagnosed until a fracture occurred. However, it is now clear that those who have had a previous low-trauma or fragility fracture are much more likely to have another. For example, adults who sustain a fracture are $50-100 \%$ more likely to have a further fracture, often within 1 year. ${ }^{14}$

Thus, it is imperative that we identify patients who are at risk of osteoporosis and fragility fractures, and ensure that they receive optimal advice and treatment to prevent first and subsequent fractures. ${ }^{15}$ At-risk patients may be identified opportunistically when visiting healthcare professionals for other reasons, or by a more systematic identification of patients from practice records. The risk factors for osteoporosis are shown in Table 1.
Table 1. Risk factors for the development of osteoporosis. ${ }^{6,} 16-18$

\begin{tabular}{|c|c|}
\hline \multirow[t]{3}{*}{ Genetic } & Female gender \\
\hline & Maternal history of hip fracture \\
\hline & Caucasian or Asian ethnicity \\
\hline \multirow[t]{3}{*}{ Demographic } & Old age ( $\geq 65$ years) \\
\hline & Low body weight (body mass index $<19 \mathrm{~kg} / \mathrm{m}^{2}$ ) \\
\hline & Previous fracture after low-energy trauma \\
\hline \multirow[t]{3}{*}{ Hormonal } & Oestrogen deficiency (early menopause, \\
\hline & hysterectomy) \\
\hline & Hypogonadism (men) \\
\hline \multirow[t]{8}{*}{ Disease } & Rheumatoid arthritis \\
\hline & Ankylosing spondylitis \\
\hline & Hyperthyroidism \\
\hline & Crohn's disease (which affects calcium \\
\hline & absorption) \\
\hline & Serious organ disease (congestive heart failure, \\
\hline & renal failure, chronic liver disease, chronic \\
\hline & pulmonary disease) \\
\hline Behavioural/ & Low calcium intake (<500-850 mg/day) \\
\hline \multirow[t]{5}{*}{ Lifestyle } & Sedentary lifestyle \\
\hline & Vitamin D deficiency \\
\hline & Smoking \\
\hline & Excessive alcohol consumption $(>4$ units \\
\hline & per day)* \\
\hline \multirow[t]{4}{*}{ Drug treatment } & Systemic glucocorticoids \\
\hline & Anticonvulsants \\
\hline & High-dose, long-term heparin \\
\hline & High-dose methotrexate as cancer therapy \\
\hline
\end{tabular}

$* 1$ unit alcohol $=8.0 \mathrm{~g}$

\section{Management}

Management of osteoporosis is multifaceted and includes:

- lifestyle changes - smoking cessation, tailored exercise programmes, and reductions in alcohol consumption can slow the rate of bone loss

- vitamin D and calcium supplementation, particularly in the elderly

- reducing risk of falls, by correcting any eye problems, and making the home environment safer 
- careful and vigilant use of glucocorticoids

- pharmacological treatments to prevent bone loss:

- SR has a dual mechanism of action, increasing bone mass by targeting bone resorption and bone formation

- parathyroid hormone promotes new bone formation by continually activating osteoclasts

- bisphosphonates, selective oestrogen receptor modulators and calcitonin inhibit bone resorption.

\section{PHARMACOLOGY}

Osteoporosis results from an imbalance in bone remodelling in which bone resorption exceeds bone formation, such that the structural integrity, and therefore strength of the bone, gradually becomes compromised.

\section{Mechanism of action}

\section{Bone turnover}

In many ways SR meets the criteria for the ideal anti-osteoporotic agent in that it has a dual mechanism of action, both decreasing bone resorption and maintaining or promoting bone formation, thereby rebalancing bone turnover in favour of bone formation. The dual mechanism of action of SR is in contrast to other therapies (alendronate, calcitonin, risedronate, raloxifene) which alter the rate of bone remodelling and lower the risk of fracture by reducing bone resorption, but do not address the reduction in bone formation that occurs within months of initiating treatment, and parathyroid hormone, which increases bone formation.

SR consists of two atoms of stable strontium and one molecule of ranelic acid, which dissociates in the gastrointestinal tract. The pharmacological actions of SR are due to strontium, which is in the same chemical group as calcium and displays a great affinity for bone; following administration, strontium is almost exclusively deposited in the skeleton.
An in vitro study in human osteoblasts demonstrated that SR promotes bone formation by stimulating preosteoblast proliferation, which is predicted to increase bone matrix synthesis. ${ }^{19,20}$ It also decreased osteoclast differentiation and activity and thus bone resorption, an event mediated by an increase in the expression of osteoprotegerin mRNA in osteoblasts. ${ }^{19}$ The net effect of these changes is reduced bone loss and increased bone mass and strength.

SR may mediate at least some of its pharmacological effects through the calciumsensing receptor expressed in osteoblasts, and in particular, influences the replication of preosteoblasts by its actions at this receptor. ${ }^{21}$ Mechanisms via other receptors are also likely to be involved. ${ }^{22}$

MicroCT analysis has shown that 3 years' treatment with SR improved the threedimensional structure of cortical and trabecular bone, which is likely to improve the biomechanical competence of bone and therefore reduce fracture risk. ${ }^{23}$

Further studies have shown that SR does not affect the degree of mineralisation; the effects of $\mathrm{SR}$ on BMD appear to result from an increase in bone mass, with strontium being deposited dosedependently into new bone structure units. Bone strontium content reaches a plateau after 2-3 years of treatment, ${ }^{24}$ but clinical improvements in fracture risk increase beyond 3 years (see below).

\section{Biochemical markers of bone turnover}

Biochemical markers for metabolic bone diseases such as osteoporosis have a role to play in clinical diagnosis and in measuring the efficacy of therapy. ${ }^{25}$ Markers for bone formation include $\mathrm{N}$-terminal propeptide of type 1 collagen (P1NP), which is produced by proliferating osteoblasts, and bone-specific alkaline phosphatase. Markers for bone resorption include the C-terminal telopeptide of type 1 collagen (CTx), which has the highest contribution from bone. ${ }^{26}$ 
The early osteoanabolic effect of SR on bone turnover was compared with teriparatide (TPTD), a recombinant form of parathyroid hormone, indicated for treatment of postmenopausal women with osteoporosis who are at high risk of fracture. In a multicentre, open label trial, 80 postmenopausal women with osteporosis were randomised to receive $20 \mathrm{mcg}$ per day teriparatide or $2 \mathrm{~g}$ per day SR. Serum biochemical markers of bone turnover were measured at baseline and then at 1, 3 and 6 months. These included P1NP and alkaline phosphatase for bone formation, and CTx for bone resorption. Also iliac bone biopsies were taken for histomorphometric analysis of cancellous, endocortical and cortical bone. Results were expressed in terms of baseline bone turnover $(\% \mathrm{MS} / \mathrm{BS})$ and mean change in bone markers at 6 months. There was no statistically significant difference in overall bone turnover between the two groups. Markers for bone formation were increased in the TPTD group with a rapid rise in P1NP (131.26\%, $\mathrm{p}<0.005$ vs baseline), whereas there was a slight decrease in the SR group $(-18.75 \%, p<0.005$ vs baseline). Markers for bone resorption (serum CTx) were reduced in the SR group $(-6.30 \%$, $p<0.005$ vs baseline) but increased in the TPTD group $(110 \%, p<0.005$ vs baseline). Indicators of cortical porosity were higher in the TPTD group. ${ }^{27}$ This study supports previous findings that SR acts predominantly as an anti-resorptive agent, thus maintaining bone structural integrity.

\section{Pharmacokinetics}

Following oral dosing, SR shows low absorption (25\% bioavailability), distribution $(1 \mathrm{~L} / \mathrm{kg})$ and plasma protein binding $(25 \%)$. It is eliminated rapidly via the kidneys and gastrointestinal tract and there is no evidence of metabolism. ${ }^{25}$

The elimination half-life of SR is 60 hours, and steady-state concentrations are reached after about 2 weeks of daily administration.
Concentrations of strontium in bone plateau after about 3 years of treatment.

SR is suitable for the elderly and for patients with hepatic impairment or mild-tomoderate renal impairment (creatinine clearance $30-70 \mathrm{~mL} / \mathrm{min}$ ) without dosage adjustment. SR is not recommended for patients with severe renal impairment (creatinine clearance below $30 \mathrm{~mL} / \mathrm{min})$.

\section{Drug interactions}

SR is not metabolised and is therefore unlikely to interact with other drugs. To date, no evidence of interaction has been detected with a number of commonly prescribed drugs (e.g. diuretics, nonsteroidal anti-inflammatory drugs [NSAIDs], cardiac glycosides, calcium-channel blockers, $\beta$-blockers, angiotensin-converting enzyme $[\mathrm{ACE}]$ inhibitors and oral anticoagulants). ${ }^{28}$

Aluminium and magnesium hydroxides decreases the absorption of SR by $20-25 \%$, and it is therefore recommended that these agents are not taken within at least 2 hours of SR administration. ${ }^{22}$ SR may also form complexes with oral tetracycline and quinolone antibiotics, thereby reducing their absorption; concomitant administration is therefore not recommended. ${ }^{28}$

\section{Dosing schedule}

The recommended dose of $\mathrm{SR}$ is $2 \mathrm{~g}$ once daily, administered orally. Once-daily dosing is preferred by almost half of patients, and is likely to improve compliance. ${ }^{29}$

The drug is supplied as granules, which should be suspended in water and consumed immediately. The bioavailability of SR is reduced by $60-70 \%$ when administered with food, particularly diary products. It is therefore recommended that $\mathrm{SR}$ is taken at bedtime, and that calcium and vitamin D supplements are taken in the morning. Food or calcium supplements should not be consumed within 2 hours of taking SR. 


\section{Correction of BMD measurements}

Strontium attenuates $\mathrm{X}$-rays to a greater extent than calcium. As a result, BMD measurements performed using dual-energy X-ray absorptiometry (DXA) will be overestimated and require correction. A $1 \%$ strontium concentration in vitro induces a $10 \%$ increase in BMD at the iliac crest which is used as a proxy for the vertebral concentration. However, the relationship between bone strontium content at the iliac crest and the hip is unclear..$^{30}$

In a theoretical study, correction ratios ranged from 9.0 to 10.8 for a variety of different DXA systems according to the effective photon energy of the X-ray beam. ${ }^{31}$ Measurements with singleenergy quantitative computed tomography (SEQCT) also require adjustment. Thus, it is important that BMD measurements in SR-treated patients are adjusted for bone strontium content, especially when interpreting DXA scans after discontinuation of SR treatment.

\section{CLINICAL EFFICACY}

\section{Stratos}

The minimum effective dose of SR was determined to be $2 \mathrm{~g}$ per day in the STRATOS (Strontium Ranelate Treatment of Osteoporosis) trial, involving 353 postmenopausal women with osteoporosis. Inclusion criteria were a lumbar $\mathrm{T}$-score below $\leq 2.4$, and at least one previous vertebral fracture. ${ }^{32}$ Here we present only the data from STRATOS relating to the clinical dose, $2 \mathrm{~g}$ daily. All women also received supplementary calcium (500 mg/day) and vitamin D (800 IU/ day).

The lumbar spine BMD increased by $2.97 \%$ per year $(p<0.01$ vs placebo) and femoral neck BMD by $3.05 \%$ per year $(p<0.01)$ over the 2 years of the study (Figure 1). During the second year of treatment, $26.5 \%$ of women taking SR experienced a new vertebral fracture, compared with $47.3 \%$ of women in the placebo group, a
Figure 1. Mean changes in baseline in lumbar and femoral neck bone mineral density over 2 years' treatment with strontium ranelate, $2 \mathrm{~g}$ daily, or placebo. $^{32}$

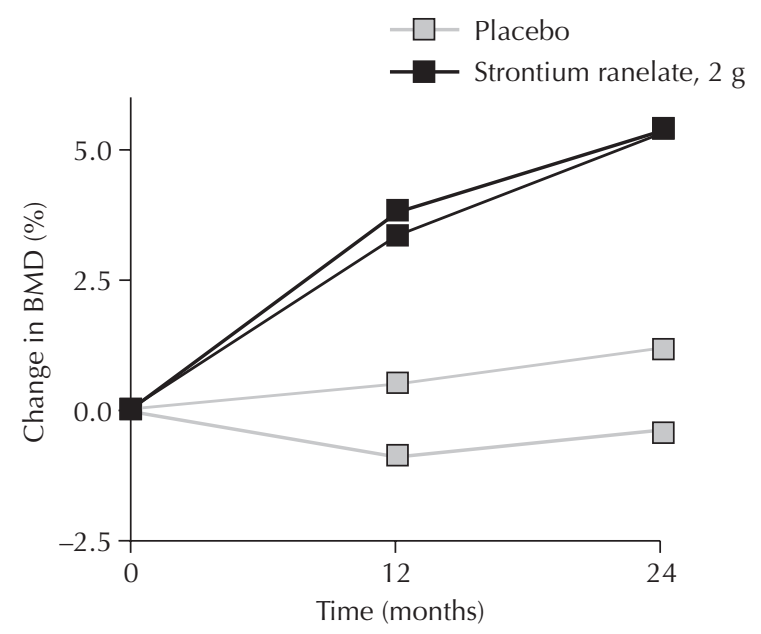

relative risk of 0.56 . The authors suggested that it takes at least 12 months for the anti-osteoporotic effect of SR to emerge.

Urinary excretion of C-terminal telopeptide of type I collagen (CTX) decreased by 20.2\% during the first 6 months of treatment with SR compared with $1.5 \%$ in the placebo group $(p<0.005)$.

\section{Phase III trials}

The efficacy of SR in reducing osteoporotic fractures has been demonstrated in two key placebo-controlled, double-blind phase III studies:

- SOTI (Spinal Osteoporosis Therapeutic Intervention)

- TROPOS (Treatment of Peripheral Osteoporosis).

The SOTI and TROPOS studies, which were carried out simultaneously in the same centres, investigated the efficacy and tolerability of SR, $2 \mathrm{~g}$ daily, to postmenopausal women with osteoporosis, with the main aim of demonstrating a reduction in the number of postmenopausal women sustaining a new osteoporotic fracture 
- either vertebral (SOTI) or non-vertebral (TROPOS) - after 4 years' (SOTI) and 5 years' (TROPOS) continuous treatment with SR. ${ }^{33}$ Figure 2 shows the rationale behind patient recruitment into either the SOTI or TROPOS studies.

\section{FIRST}

All participants in the SOTI and TROPOS studies took calcium (up to $1 \mathrm{~g}$ ) and vitamin D (400-800 IU) supplements for 2-24 weeks before entering the double-blind treatment phase - the Fracture International Run In for Strontium Ranelate Trials (FIRST) - in an attempt to normalise calcium and vitamin D status before starting the main trial. These supplements were continued throughout the double-blind treatment period in both studies.

Figure 2. Decision flowchart for the inclusion of patients in either the Spinal Osteoporosis Therapeutic Intervention (SOTI) or Treatment of Peripheral Osteoporosis (TROPOS) clinical trials. ${ }^{33}$

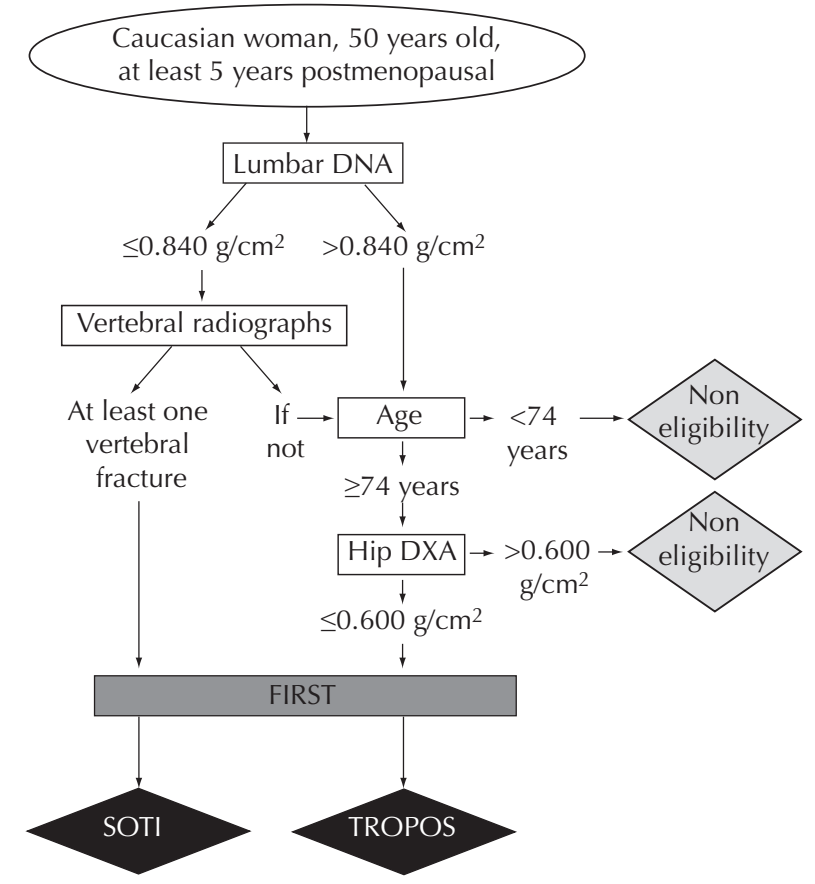

DXA, dual-energy X-ray absorptiometry; FIRST, Fracture International Run In for Strontium Ranelate Trials.

\section{SOTI}

The SOTI study enrolled 1640 postmenopausal women with osteoporosis and at least one vertebral fracture. ${ }^{34}$ After 1 year of treatment, the rate of new vertebral fractures was significantly lower in the SR group than in the placebo group (6.4 vs $12.2 \%$; relative risk [RR] 0.51 ; number needed to treat $[\mathrm{NNT}] 17 ; p<0.001)$. After 3 years, the rate was 20.9 and $32.8 \%$, respectively - a risk reduction of $41 \%$ (RR 0.59, NNT 9; $p<0.001)$.

At 3 years, non-adjusted BMD in the lumbar spine, femoral neck and total hip increased by $12.7 \%, 7.2 \%$ and $8.6 \%$, respectively, from baseline. These increases were significantly greater than in the placebo group (differences of $14.4,8.3$ and $9.8 \%$ for the lumbar spine, femoral neck and total hip, respectively; $p<0.001)$. The difference between the SR and placebo groups remained significant when lumbar spine BMD was adjusted for bone strontium content (6.8 vs $1.3 \% ; p<0.001)$.

From 3 months onwards, serum concentrations of bone-specific alkaline phosphatase (BSAP) in the SR group were significantly greater than in the placebo group, and CTX levels were significantly lower $(p<0.05)$.

The efficacy of SR on vertebral fracture was sustained over 4 years, with a reported $33 \%$ reduction in vertebral fracture risk in the SR group compared with the placebo group $(p<0.001)$.

Analysis of data from SOTI as part of a Cochrane systematic review demonstrated a 52\% relative reduction in symptomatic or clinical vertebral fractures over 1 year and 38\% relative reduction over 3 years (RR 0.48, 95\% CI 0.29 to 0.80 at 1 year; RR $0.62,95 \%$ CI 0.47 to 0.83 at 3 years). ${ }^{2}$ Overall, the reduction in the relative risk of vertebral fractures was considered to be similar to the $40-60 \%$ reported with other therapies. ${ }^{35}$

\section{TROPOS}

TROPOS is the longest placebo-controlled clinical trial ever conducted in the field of osteoporosis 
management. It examined the effect of long-term (5 years') treatment with SR on vertebral and nonvertebral fracture risk in 5091 postmenopausal women with osteoporosis. ${ }^{36}$ The study population was heterogeneous, and $29.9 \%$ of women had a prevalent fragility fracture at baseline.

Over 3 years, treatment with SR was associated with a $16 \%$ reduction in the relative risk for non-vertebral fracture compared with placebo (NNT 55; $p=0.04$ ). The risk of sustaining a non-vertebral fragility fracture (i.e. hip, wrist, pelvis, sacrum, ribs) was 19\% lower in the SR group than in the placebo group (NNT $56 ; p=0.31$ ).

In a subgroup of women designated as being at high risk of such fractures because of their age (74 years or older) and femoral neck BMD (T-score $\leq 3 \mathrm{SD}$ from normal), the relative risk of hip fracture was reduced by $36 \%$ with SR (NNT 48; $p=0.046)$.

Preliminary reporting of the 5-year data showed a reduction in relative risk of vertebral fracture of $24 \%(\mathrm{RR} 0.76 ; p<0.001)$ and a $15 \%$ risk reduction in non-vertebral fracture. ${ }^{37}$ In the high risk subgroup of women (mean age 79.2, mean lumbar $\mathrm{T}$-score $\leq 4.2$, mean femoral neck T-score $\leq 3.0$ ), the hip antifracture efficacy of SR was assessed at 5 years and demonstrated a $43 \%$ reduction in relative risk of hip fracture (RR 0.57; p=0.036). ${ }^{38}$ Thus, SR is the first antiosteoporotic treatment that has been shown to reduce vertebral and non-vertebral fracture risk, also hip fracture risk in a high risk group, over 5 years' treatment.

Of those patients undergoing annual spinal X-ray ( $\mathrm{n}=3640)$, the risk of sustaining a new vertebral fracture was reduced by $45 \%$ over 1 year and 39\% over 3 years with SR compared with placebo ( $p<0.001$ for both comparisons). In women who had no vertebral fracture at baseline $(n=2416)$, the risk of a first vertebral fracture was reduced by $45 \%$ following treatment with SR $(p<0.001)$.
Long-term treatment with SR was associated with a significant increase from baseline in nonadjusted BMD at the femoral neck and total hip (3-year increases of 5.7 and $7.1 \%$, respectively; $p<0.001$ vs baseline). At 3 years, the corresponding differences between the SR and placebo groups were 8.2 and $9.8 \%$ for femoral neck and total hip, respectively $(p<0.001$ for both comparisons vs placebo).

\section{Pooled analyses}

Similar designs were used for the SOTI and TROPOS studies so that the data could be pooled to provide a larger dataset, thus enabling more robust conclusions to be drawn. Post hoc analyses of pooled data from the SOTI and TROPOS studies showed that treatment-mediated increases in BMD over 3 years' treatment were associated with a reduced incidence of new clinical vertebral fractures. ${ }^{39,40}$ Each $1 \%$ increase in BMD at the femoral neck was associated with a $4 \%$ decrease in the risk of ew clinical vertebral fracture ${ }^{39}$ and a 3\% decrease (95\% CI 1-5\%) in new vertebral fracture. ${ }^{40}$ Each $1 \%$ decrease in total proximal femur BMD was associated with a $2 \%$ decrease (95\% CI 1-4\%) in risk of new vertebral fractures. The 3-year changes in femoral neck and total proximal femur BMD explained 76 and $74 \%$, respectively, of the reduction in vertebral fractures observed with treatment with SR. An increase in femoral neck BMD at 1 year was significantly associated with the reduction in incidence of new vertebral fractures after 3 years $(p=0.04)$. Three-year changes in spine BMD were not associated with the incidence of new vertebral fracture, but a trend was found for femoral neck BMD $(p=0.09)$ and total proximal femur BMD $(p=0.07){ }^{40}$

Women experiencing new clinical vertebral fractures gained less BMD $(3.58 \%)$ at the femoral neck than those without new vertebral fractures $(5.69 \% ; p<0.01)$. Similarly, women who 
experienced a hip fracture gained less BMD at the femoral neck than those without hip fracture (3.4 vs $7.2 \%$; $p=0.02$ ). Furthermore, increases in BMD at the femoral neck and total hip at 1 year induced by SR were predictive of a reduction in the incidence of new clinical vertebral fractures after 3 years' treatment. ${ }^{41}$

Finally, using the pooled data set from the SOTI and TROPOS studies, the beneficial effects of treatment with SR were shown to be independent of age, initial $\mathrm{BMD}$, prevalent vertebral fracture, familial history of osteoporosis, baseline body mass index and smoking status. ${ }^{42}$

The Cochrane Collaboration has conducted a systematic review of data from the STRATOS, SOTI and TROPOS trials together with data from the PREVOS study, an early trial of $1 \mathrm{~g}$ SR daily for the prevention of early postmenopausal bone loss. ${ }^{43}$ The review concluded that there was silver-level evidence to support the efficacy of SR in reducing vertebral fractures in postmenopausal women with osteoporosis and, to a lesser extent, non-vertebral fractures, and in increasing BMD in postmenopausal women with and without osteoporosis. $^{2}$ In the treatment population (STRATOS, SOTI and TROPOS; 5254 patients), there was a $41 \%$ relative reduction in radiographic vertebral fractures over a 1 year period and a $37 \%$ relative reduction over 3 years (SOTI and TROPOS; 5082 patients) compared with placebo (RR $0.59,95 \%$ CI 0.46 to 0.74 , NNT 32 at 1 year; RR $0.63,95 \%$ CI 0.56 to 0.71 , NNT 13 at 3 years).

In terms of non-vertebral fractures, data from the SOTI and TROPOS studies $(\mathrm{n}=6572)$ demonstrated a $14 \%$ reduction over 3 years with 2 g SR daily compared with placebo (RR 0.86 $95 \%$ CI 0.75 to 0.98 , NNT 58 ).

\section{SOTI/TROPOS extension study}

Both the SOTI and TROPOS trials were extended by a 3-year open label extension study to assess the efficacy of $2 \mathrm{~g}$ /day SR treatment over 8 years. Only patients who had received SR treatment continuously during the 8 year period were included in the analysis. The lumbar and femoral neck BMD increased continuously in patients treated with strontium ranelate over 8 years, with a mean increase of $4.47 \%$ in lumbar BMD which remained significant throughout the entire 8-year period $(p<0.001)$ (Figure 3). The incidence of vertebral and non-vertebral fractures over the 3-year extension period $(13.7 \%)$ was similar to the incidence observed in SOTI and TROPOS at 3 years (11.5\%), and was not significantly different $(p=0.94)$. These results confirm a sustained efficacy of strontium ranelate over time, despite an increased risk with aging. The safety profile over 8 years was in agreement with previous findings. ${ }^{44}$

\section{Efficacy in spinal osteoarthritis}

A post-hoc analysis of a subgroup of patients from the SOTI and TROPO studies was carried out to determine whether treatment with SR for a 3-year period was effective in delaying the progression of clinical and structural osteoarthritis (OA). Patients included in this analysis were 1105 women with osteoporosis and

Figure 3. Continuous increase in BMD over 8 years at lumbar site and at femoral neck site (taken from Reginster JY et al. ${ }^{44}$ )

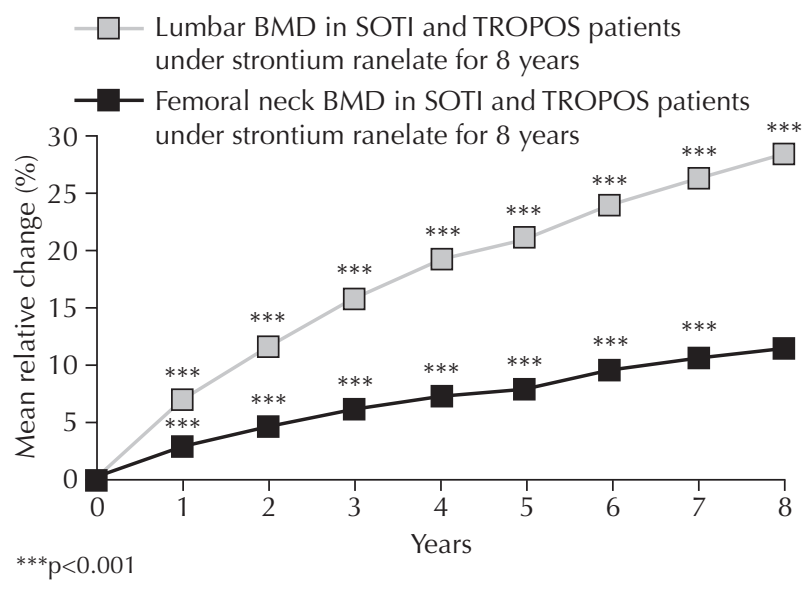


concomitant spinal $\mathrm{OA}$ and for whom lumbar $\mathrm{X}$-rays were available at baseline and during the 3-year treatment period. Progression of spinal OA was measured by the presence and severity of osteophytes, an indicator of lumbar disk degeneration, disc space narrowing and sclerosis in the lumbar intervertebral spaces. Results were graded to provide an overall OA score for each intervertebral space. Back pain and quality of life were also assessed. After 3 years only 9.9\% patients in the SR group suffered a progression in the overall OA score compared to $17.1 \%$ in the placebo group, and the proportion of patients with an increase in the overall spinal OA score was reduced by $42 \%$ in the SR group compared with placebo $(\mathrm{RR} 0.58, p=0.0005)$. More patients in the SR group experienced an improvement in back pain compared with placebo, although no overall difference in quality of life was observed. ${ }^{45}$ Although the mechanism of action of SR in reducing the progression of spinal $\mathrm{OA}$ is not known, previous studies have indicated that in addition to its effect on bone, SR also has a direct effect on cartilage ${ }^{46,47}$ to promote endochondral ossification.

\section{Quality of life}

Quality of life was assessed in the SOTI study using the Quality of Life questionnaire in Osteoporosis (QUALIOST), which women completed at baseline and every 6 months throughout the trial. The QUALIOST is a 23-item diseasespecific (vertebral osteoporosis) questionnaire with a global score and emotional and physical subscores. Data from a subset of 1240 women, reported in abstract form, showed that, compared with placebo, global, emotional and physical scores were all significantly reduced after 3 years' treatment with $\mathrm{SR}(p=0.016, p=0.019, p=0.032$, respectively), indicating that $\mathrm{SR}$ has a beneficial effect on quality of life. ${ }^{48}$ Furthermore, results from the back pain assessment included in the QUALIOST showed a significant improvement in back pain from the first year $(p=0.006)$ and a reduction of $29 \%$ with SR compared with the placebo group at 3 years. ${ }^{48}$

\section{Efficacy in the elderly}

The very elderly ( $\geq 80$ years) are at a particularly high risk of all types of fracture and, in particular, non-vertebral fractures such as those of the hip, humerus and wrist, partly because of decreased bone strength but also because of increased propensity to falls, resulting from impaired vision, poor balance and lower muscle mass compared with younger people. ${ }^{4,49}$ Indeed, 60\% of all hip fractures occur in this population. ${ }^{50}$ Data from the SOTI and TROPOS studies were evaluated in a pre-planned retrospective analysis to examine the efficacy of SR in terms of its effects on fracture risk in a population of 1488 women aged 80 years and over (mean age: 83.5 years; mean lumbar BMD T-score: -2.7 ; mean femoral neck BMD T-score: -3.3$).^{51}$

Treatment with SR was associated with an early and sustained reduction in both vertebral and non-vertebral fracture. Thus, after 1 year the risk of vertebral fracture and clinical vertebral fracture was reduced by $59 \% \quad(p=0.002)$ and $37 \% \quad(p=0.002)$, respectively, compared with placebo. ${ }^{51}$ The risk of non-vertebral fracture was reduced by $41 \% \quad(p=0.0027)$. These reductions in fracture risk at 1 year were sustained with continuing treatment over 3 years. Corresponding risk reductions at 3 years were $32 \%(p=0.013)$, $22 \%(p=0.04)$ and $31 \%(p=0.011)$.

Recent data from the SOTI and TROPOS trials suggest that the effects on fracture risk are maintained for up to 5 years of continuous SR treatment, the risk of vertebral and nonvertebral fracture being reduced by $31 \%$ and $26 \%$, respectively, compared with placebo. ${ }^{52}$

SR is the only antiosteoporotic drug that has been shown to achieve an early and sustained risk reduction (up to 5 years) in vertebral and nonvertebral fracture in such an elderly population 
$\left(\geq 80\right.$ years). ${ }^{52}$ These findings are particularly relevant given that the frequency and complications of fractures increase with advancing age. In practice, osteoporosis is often not diagnosed or is treated suboptimally in elderly patients. Indeed, a recent report by the Information Centre for Health and Social Care on standards of care for osteoporosis and falls in primary care showed that more than a quarter of older women with diagnosed osteoporosis and a previous fracture still do not receive appropriate drug treatment. ${ }^{8}$

$\mathrm{SR}$ is much easier to take than the bisphosphonates, and does not have any known interactions with a wide range of drugs likely to be coadministered in the elderly. Thus, SR is clearly a useful drug for the treatment of this at-risk population.

\section{Efficacy in younger postmenopausal women with severe osteoporosis}

Early fractures in the first 10 years after menopause can have a major impact on the progression and impact of osteoporosis, and it has been well established that initial fractures at any skeletal site can significantly increase the risk of a future additional fracture. Therefore, early and sustained antiosteoporotic treatment has the potential to reduce this ongoing fracture risk in these women.

An analysis of a group of younger postmenopausal women (aged 50-65 years; mean age 60 years) enrolled in the SOTI trial $(n=353)$ was conducted to assess the impact of early intervention with strontium ranelate on fracture risk. ${ }^{53}$ In addition, these younger women had severe osteoporosis by virtue of their low BMD T-scores at the lumbar spine (mean T-score: -3.6$)$ and the femoral neck (-2.5). More than $80 \%$ of these women also had a prevalent vertebral fracture at baseline, with nearly onequarter having prevalent non-vertebral fractures. After 3 years of strontium ranelate treatment the risk of vertebral fracture was reduced by $47 \%$
( $\mathrm{RR} 0.53 ; p=0.006)$. This reduction in fracture risk was accompanied by a parallel increase in BMD at the lumbar spine $(13.7 \% ; p<0.001)$ and femoral neck (7.5\%; no $p$-value reported). The magnitude of the antifracture efficacy of strontium ranelate in this younger population was similar to that reported in the entire SOTI patient population. Thus, in summary, strontium ranelate has significant antifracture efficacy in younger women under 65 years of age with severe osteoporosis and appears to have utility in reducing fracture risk regardless of the age of postmenopausal women.

\section{SAFETY AND TOLERABILITY}

Given that anti-osteoporotic medications are taken for many years and do not produce symptomatic improvement in the condition, it is important that drugs cause minimal side effects, otherwise many patients simply will not take them.

Safety data derived from controlled clinical trials performed to date have shown SR to be well tolerated, exhibiting a similar adverse event profile to that of placebo. Table 2 shows the adverse events reported most commonly during the phase 3 placebo-controlled clinical trials of SR. During the SOTI and TROPOS studies, diarrhoea, nausea, headache and eczema/

Table 2. The most common* adverse events reported during phase 3 clinical trials of strontium ranelate $(\mathrm{n}=6669) \cdot{ }^{34,36}$

\begin{tabular}{ll}
\hline $\begin{array}{l}\text { Adverse event } \\
\text { placebo) }\end{array}$ & Frequency (vs \\
\hline $\begin{array}{l}\text { Nervous system disorders } \\
\text { Headache }\end{array}$ & 3.0 vs $2.4 \%$ \\
Gastrointestinal & \\
Nausea & 6.6 vs $4.3 \%$ \\
Diarrhoea & 6.5 vs $4.6 \%$ \\
Loose stools & 1.1 vs $0.2 \%$ \\
Skin and subcutaneous tissue disorders & \\
Dermatitis & 2.1 vs $1.6 \%$ \\
Eczema & 1.5 vs $1.2 \%$ \\
\hline
\end{tabular}

*Common, $>1 / 100$ and $<1 / 10$ patients. 
dermatitis were reported more frequently in the SR treatment groups than in placebo group over the first 3 months of administration, although such differences tended to resolve with continued treatment. ${ }^{34,36}$ The safety profile of SR in a very elderly population (aged $\geq 80$ years) was similar to that in younger patients, as demonstrated by a subgroup analysis of this population from the SOTI and TROPOS studies. ${ }^{51}$

The Cochrane systematic review pooled data on adverse events from the STRATOS, SOTI and TROPOS trials $(\mathrm{n}=6847) .{ }^{2}$ There was no significant difference in risk of withdrawal in SR-treated patients compared with placebo recipients. In terms of withdrawal due to adverse events, 22\% of SR-treated patients and 19.1\% of placebo-recipients withdrew and the number of emergent adverse effects was also similar. Serious adverse events occurred in $24.09 \%$ of SR-treated patients and $23.97 \%$ of placebo recipients (difference not significant).

Overall, diarrhoea occurred in $6.5 \%$ of SR-treated patients and $4.7 \%$ of placebo recipients. Gastritis occurred in 2.7\% of SR-treated patients and $3.4 \%$ of placebo recipients.

Additional data obtained suggested that the risk of vascular system disorders including venous thromboembolism and pulmonary embolism was slightly increased with taking $2 \mathrm{~g}$ SR daily for 3-4 years compared with placebo (two trials, $\mathrm{n}=6669$; venous thromboembolism: $2.2 \%$ vs $1.5 \%$, OR $1.5,95 \%$ CI 1.1 to 2.1 ; pulmonary embolism: $0.8 \%$ vs $0.4 \%$, OR $1.7,95 \%$ CI 1.0 to 3.1). The risk of nervous system disorders such as headaches $(3.9 \%$ vs $2.9 \%)$, seizures $(0.3 \%$ vs $0.1 \%)$, memory loss $(2.4 \%$ vs $1.9 \%)$ and disturbance in consciousness $(2.5 \%$ vs $2.0 \%$ ) was also slightly increased.

Mean baseline serum creatine kinase levels increased in both groups but the increase was significantly greater in the SR group (31.3 \pm 80.8 IU/L) than controls (13.1 $\pm 46.6 \mathrm{IU} / \mathrm{L})$; estimated difference $=18.2 \mathrm{IU} / \mathrm{L}(95 \%$ CI 14.8 to 21.6$)$. The serum creatine kinase levels were greater than the upper limit of normal on at least one occasion in $29.4 \%(789 / 2680)$ of the SR group and $17.6 \%$ (475/2705) of the controls (RR 1.68, 95\% CI 1.52 to 1.85), providing evidence that $\mathrm{SR}$ may affect skeletal muscle cell integrity and that targeted surveillance is warranted. Further exploration and quantification of these adverse effects will be useful.

The manufacturer recommends that $\mathrm{SR}$ is not given to patients with severe renal impairment, and, in line with good clinical practice, advocates periodic assessment of renal function in patients with chronic renal impairment. ${ }^{28} \mathrm{SR}$ should be used with caution in patients at increased risk of venous thromboembolism, including patients with a history of thromboembolic events. The annual incidence of venous thromboembolism reported during phase 3 studies was $0.7 \%$ over 4 years, with a relative risk of 1.42 in patients taking SR compared with placebo recipients.

\section{Compliance}

Although compliance in the SOTI and TROPOS trials was relatively high at $80-93 \%,{ }^{2}$ compliance with anti-osteoporotic medications in the clinic tends to be poor, particularly with daily and weekly formulations ${ }^{54}$ - as with many essentially 'silent' conditions in which a patient generally feels well. Some patients find that the bisphosphonates are particularly inconvenient to take, as they are required to remain upright for at least 30 minutes after taking the medication and cannot eat or drink during this period.

A recent study of 2485 people $(90.3 \%$ women) over 55 years of age showed that almost half $(45 \%)$ preferred to take anti-osteoporotic medications once daily rather than once weekly or once monthly. ${ }^{29}$ Furthermore, given the choice between three different medication regimens, those not currently using anti-osteoporotic medication preferred a theoretical medication that was daily and did not involve subsequent fasting and maintaining an upright posture, as 
is required with the bisphosphonates. Thus, compliance with anti-osteoporotic medications could be improved if patients are able to choose a therapeutic regimen best suited to their particular needs. ${ }^{29} \mathrm{SR}$ is likely to be preferred by a majority of patients with osteoporosis given its convenient once-daily administration.

\section{Cost-effectiveness}

Borgström et al ${ }^{55}$ used a Markov cohort model populated with Swedish cost and epidemiological data, and with a societal perspective, to estimate cost-effectiveness for two populations corresponding to the two populations in the SOTI and TROPOS trials. Thus, for 69-year-old women with low BMD and vertebral fractures (equivalent to patients in the SOTI study) in the base case analysis, the cost per quality-adjusted life years (QALY) gained for treatment with SR compared with no treatment was estimated at SEK472,686 $(€ 50,496)$ (SOTI) when costs in added life years were included, and SEK336,420 (€35,950) when these latter costs were excluded. For 77-year old women with low BMD (TROPOS) the costs were SEK259,643 (€27,743) and SEK165,680 $(€ 17,710)$, respectively. Thus the analysis showed that, compared with no treatment, SR is costeffective in the treatment of postmenopausal women with low BMD.

Stevenson et $a l^{10}$, in a Health Technology Assessment, used an updated version of the Sheffield Health Economic Model for Osteoporosis populated with absolute risk of fractures using an algorithm being developed for the World Health Organization and supplied in confidence to the authors. SR provided gains in QALYs compared with no treatment in women with sufficient calcium and vitamin $\mathrm{D}$ intake. The size of the QALY gain for each intervention was strongly related to the absolute risk of fracture. They concluded that SR is cost-effective for the treatment of women at relatively high risk of osteoporotic fracture.
A probabilistic sensitivity analysis, using efficacy data from the randomised controlled trials, suggested that SR may be less cost-effective than alendronate. However, this analysis had some limitations, in terms of analysing the cost-effectiveness of anti-osteoporotic agents in general, and SR in particular. The authors point out that the evidence base for the T-score by age of the general female population needs to be strengthened, particularly in women over the age of 80 years, and that the prevalence of risk factors associated with fracture rates, over and above that provided by BMD, also needs to be significantly strengthened to ensure that the estimated number of women that could be costeffectively treated is accurate.

Furthermore, studies in the USA have shown that only $24 \%$ of patients taking bisphosphonates and $18 \%$ of patients taking raloxifene were still taking their medication at 1 year. ${ }^{56}$ Data on compliance and continuance with SR outside of clinical trials are not yet available, but the easier dosing with SR and lack of gastritis in particular are likely to be key factors that improve continuance with SR compared with the bisphosphonates, and may well increase the apparent cost-effectiveness of this agent in the long run.

The time-horizon of the model was, by necessity, restricted to 10 years. ${ }^{10}$ However, as the time course over which younger women are likely to take anti-osteoporotic medications for primary prevention is likely to be longer than this, there is a need to examine cost-effective over a longer period of time.

Clearly, analysis of the cost-effectiveness of the treatment of osteoporosis is highly complex. However, at a simplistic level, if one considers that all the anti-osteoporotic medications reduce fracture risk to a similar extent when taken under controlled clinical trial conditions, one can expect differences in cost-effectiveness between the various agents to be affected largely by factors such as tolerability, compliance, quality 
of life and drug cost. Alendronate is the only anti-osteoporotic medication that is available as a generic formulation and it is therefore perhaps inevitable that it will appear to be more costeffective than the proprietary agents. One must also bear in mind that the body of evidence relating to long-term tolerability and concordance with drugs such as the bisphosphonates is welldeveloped, whereas this is not the case with newer agents; it is these factors, combined with long-term efficacy in reducing fracture risk that will be critical in determining the true long-term cost-effectiveness of the newer agents.

Persistence with treatment in patients with osteoporosis is generally low, ${ }^{10}$ and clearly a drug that is not taken cannot be effective. Given that patient preference in terms of tolerability and dosing regimen may improve compliance, ${ }^{23}$ it is appropriate to discuss these issues with patients before selecting a drug treatment, in order to increase the likelihood of achieving clinical benefit.

\section{KEY POINTS}

- Strontium ranelate is an anti-osteoporotic agent with an innovative mechanism of action, and is indicated for the treatment of postmenopausal osteoporosis to reduce the risk of vertebral and hip fractures.

- By promoting bone formation and reducing bone resorption, strontium ranelate uncouples the bone remodelling process in a favourable manner.

- Compared with placebo, strontium ranelate significantly increases BMD in postmenopausal women with osteoporosis and may also reduce progression of spinal OA..

- Postmenopausal women with osteoporosis had a significantly reduced risk of vertebral and non-vertebral fracture following 5 years of continuous treatment with strontium ranelate. This effect is sustained up to 8 years, despite an increased risk with aging.

- The risk of vertebral and non-vertebral fractures is significantly reduced in very elderly women $(\geq 80$ years) with osteoporosis

- Strontium ranelate is well tolerated, with an adverse event profile closely resembling that of placebo. Gastrointestinal effects, including diarrhoea, are mild-to-moderate in severity and tend to resolve during the first 3 months of treatment.

- As strontium ranelate is a divalent cation it is not metabolised. Therefore, it has limited potential for interaction with other commonly administered drugs. 


\section{REFERENCES}

1 Kai M, Anderson M, Lau E. Exercise interventions: defusing the world's osteoporosis time bomb. Bull World Health Organ 2003; 81: 827-30.

2 O'Donnell S, Cranney A, Wells GA et al. Strontium ranelate for preventing and treating postmenopausal osteoporosis. Cochrane Database Syst Rev 2006; 4: CD005326.

3 National Osteoporosis Society. What is osteoporosis? www.nos.org.uk

4 Cummings S, Melton L. Epidemiology and outcomes of osteoporotic fractures. Lancet 2002; 359: 1761-7.

5 Hauselmann HJ, Rizzoli R. A comprehensive review of treatments for postmenopausal osteoporosis. Osteoporos Int 2003; 14: 2-12.

6 Woolf A, Pfleger B. Burden of major musculoskeletal conditions. Bull World Health Organ 2003; 81: 646-56.

7 Jalava T, Sarna S, Pylkkanen L et al. Association between vertebral fracture and increased mortality in osteoporotic patients. F Bone Miner Res 2003; 18: 1254-60.

8 Information Centre for Health and Social Care. Evaluation of standards of care for osteoporosis and falls in primary care. August 31, 2007. www.ic.nhs.uk/statistics-and-datacollections/primary-care/general-practice/evaluation-ofstandards-of-care-for-osteoporosis-and-falls-in-primary-care.

9 Bailey K, Majeed A. Trends in the treatment of osteoporosis and types of drug treatment prescribed in general practice in England and Wales. 1994-1998. Health Statistics Quarterly: National Statistics Office, 2002.

10 Stevenson M, Davis S, Lloyd-Jones M, Beverley C. The clinical effectiveness and cost-effectiveness of strontium ranelate for the prevention of osteoporotic fragility fractures in postmenopausal women. Health Technology Assessment 2007; Vol. 11: No. 4.

11 World Health Organization. Prevention and management of osteoporosis. WHO Technical Report Series no. 921, 2003.

12 Compston J, Rosen C. Fast Facts - Osteoporosis. 3rd edn. Oxford: Health Press, 2002.

13 Royal College of Physicians and Bone and Tooth Society for Great Britain. Osteoporosis - Clinical guidelines for prevention and treatment. London: Royal College of Physicians, 2005.

14 Klotzbuecher C, Ross P, Landsman P et al. Patients with prior fractures have an increased risk of future fractures: a summary of the literature and statistical synthesis. 7 Bone Miner Res 2000; 15: 721-39.

15 Brown P. Osteoporosis - Disease Overview. Drugs in Context 2005; 1(15): 787-95.

16 Mattens MG. Risk of fracture and treatment to prevent osteoporosis-related fracture in postmenopausal women. A review. 7 Reprod Med 2003: 48: 425-34.

17 Hansen LN, Vondracek SF. Prevention and treatment of nonpostmenopausal osteoporosis. Am 7 Health Syst Pharm 2004; 61: 2637-56.

18 National Institute for Health and Clinical Excellence. Alendronate, etidronate, risedronate, raloxifene and strontium ranelate for the primary prevention of osteoporotic fragility fractures in postmenopausal women. June 2007. www.nice.org.uk
19 Brennan TC, Rybchyn MS, Conigrave AD, Mason RS. Strontium ranelate effect on proliferation and OPG expression in osteoblasts. Calcif Tissue Int 2006; 78 (Suppl 1): S129.

20 Reginster JY, Malaise O, Neuprez A, Bruyere O. Strontium ranelate in the prevention of osteoporotic fractures. Int 7 Clin Pract 2007; 61: 324-8.

21 Fromigué O, Barbara A, Hay E et al. Strontium ranelate stimulates murine osteoblast replication independently of calcium sensing receptor-mediated ERK1/2 activation. Osteoporosis Int 2006; 17 (Suppl 2): S227-8.

22 Marie PJ. Strontium ranelate: a dual mode of action rebalancing bone turnover in favour of bone formation. Curr Opin Rheumatol 2006; 18 (Suppl 1): S11-15.

23 Jiang Y, Zhao JJ, Genant HK. Effects of strontium ranelate on $3 \mathrm{D}$ cortical and trabecular microstructure in postmenopausal osteoporosis in multicenter, double-blind, and placebo controlled studies. Osteoporosis Int 2006; 18: 2 (Late Breaking News).

24 Boivin G, Farlay D, Simi C, Meunier PJ. Bone strontium distribution and degree of mineralisation of bone in postmenopausal osteoporotic women treated with strontium ranelate for 2 and 3 years. Osteoporosis Int 2006; 17 (Suppl 2) S86.

25 Seibel M. Biochemical Markers of Bone Turnover Part II: Clinical Applications in the Management of Osteoporosis. Clin Biochem Rev 27 2006; 27: 123-38.

26 Seibel MJ. Biochemical Markers of Bone Turnover.Part I: Biochemistry and Variability. Clin Biochem Rev 2006; 26: 97-122.

27 Recker RR, Stepan J, de la Pena P et al. Comparative effects of teriparatide and strontium ranelate on biochemical bone markers and iliac crest biopsies in postmenopausal women with osteoporosis. The 35th European Symposium on Calcified tissues 2008;

28 Servier Laboratories Limited. Protelos ${ }^{\circledR}$. Summary of product characteristics. Slough, 2004.

29 Richards JB, Cherkas LF, Spector TD. An analysis of which anti-osteoporosis therapeutic regimen would improve compliance in a population of elderly adults. Curr Med Res Opin 2007; 23: 293-9.

30 Nielsen S, Slosman D, Sorensen O et al. Influence of strontium on bone mineral density and bone mineral content measurements by dual X-ray absorptiometry. 7 Clin Densitom 1999; 2: 371-9.

31 Blake GM, Fogelman I. Effect of bone strontium on BMD measurements. $f$ Clin Densitom 2007; 10: 34-8. Epub Nov 28 2006.

32 Meunier P, Slosman D, Delmas P et al. Strontium ranelate: dose dependent effects in established postmenopausal vertebral osteoporosis - a 2-year randomized placebo controlled trial. $\mathcal{f}$ Clin Endocrinol Metab 2002; 87: 2060-6.

33 Meunier PP, Reginster J. Design and methodology of the Phase 3 trials for the clinical development of strontium ranelate in the treatment of women with postmenopausal osteoporosis. Osteoporosis Int 2003; 14: S66-76.

34 Meunier P, Roux C, Seeman E et al. The effects of strontium ranelate on the risk of vertebral fracture in women with postmenopausal osteoporosis. $\mathcal{N}$ Engl $\mathcal{J}$ Med 2004; 350: 459-68. 


\section{DRUG PROFILE}

35 Cranney A, Guyatt G, Griffith L et al. Meta-analyses of therapies for postmenopausal osteoporosis. IX: Summary of meta-analyses of therapies for postmenopausal osteoporosis. Endocr Rev 2002; 23: 570-8.

36 Reginster J, Seeman E, Vernejoul M et al. Strontium ranelate reduces the risk of nonvertebral fractures in postmenopausal women with osteoporosis: TROPOS study. f Clin Endocrinol Metab 2005; 90: 2816-22.

37 Reginster JY, Meunier PJ, Roux C et al. Strontium ranelate: an anti-osteoporotic treatment demonstrated vertebral and nonvertebral anti fracture efficacy over 5 years in post menopausal osteoporotic women. Osteoporosis Int 2006; 17 (Suppl 2): S14.

38. Reginster JY, Brixen K, Cormier C, Cannata J. Strontium ranelate demonstrates vertebral and non-vertebral anti fracture efficacy including hip fractures over 5 years in post menopausal osteoporotic women. Osteoporos Int. 2007;18: (suppl 1): S21.

39 Bruyère $\mathrm{O}$, Reginster JY. Relationship between increase in femoral neck bone mineral density and decrease in vertebral and hip fracture incidence during treatment with strontium ranelate. Osteoporosis Int 2006; 18: 7 (Late Breaking News).

40 Bruyère $\mathrm{O}$, Roux $\mathrm{C}$, Detilleux $\mathrm{J}$ et al. Relationship between bone mineral density and fracture risk reduction in patients treated with strontium ranelate. 7 Clin Endocrinol Metab 2007; 92: 3076-81.

41 Bruyère $\mathrm{O}$, Fardellone $\mathrm{P}$, Kaufman JM et al. One-year increase in hip (hip or femoral neck) bone mineral density predicts long-term (3 years) decrease in vertebral fracture incidence in patients treated with strontium ranelate. Osteoporosis Int 2006; 17 (Suppl 1): S96.

42 Roux C, Reginster JY, Fechtenbaum J et al. Vertebral fracture risk reduction with strontium ranelate in women with postmenopausal osteoporosis is independent of baseline risk factors. 7 Bone Miner Res 2006; 21: 536-42.

43 Reginster JY, Deroisy R, Dougados M et al. Prevention of early postmenopausal bone loss by strontium ranelate: the randomized, two-year, double-masked, dose-ranging, placebocontrolled PREVOS trial. Osteoporos Int 2002; 13: 925-31.

44. Reginster JY, Sawicki A, .Roces-Varela A, et al. Strontium ranelate : 8 years efficacy on vertebral and nonvertebral fractures in post menopausal osteoporotic women. Eighth European Congress on Clinical and Economic aspects of Osteoporosis (ECCEO) Istanbul 2008;

45. Bruyere O, Delferriere D, Roux C, et al. Effects of strontium ranelate on spinal osteoarthritis progression. Ann Rheum Dis 2008;67:335-9.
46. Henrotin Y, Labasse A, Zheng SX, et al. Strontium ranelate increases cartilage matrix formation. 7 Bone Miner Res 2001; 16: 299-308

47. Alexandersen M, Karsdal P, Qvist J, et al. Strontium ranelate reduces the urinary level of cartilage degradation biomarker CTX-II in postmenopausal women. Bone 2007; 40: 218-22.

48 Marquis P, De La Loge C, Diaz-Curiel M et al. Beneficial effects of strontium ranelate on the quality of life in patients with vertebral osteoporosis (SOTI Study). Osteoporosis Int. 2005; 16 (Suppl 3): S54 (P223).

49 Ettinger MP. Aging bone and osteoporosis: strategies for preventing fractures in the elderly. Arch Intern Med 2003; 163: 2237-46.

50 Mueller G, Russell R. Osteoporosis: pathogenesis and clinical intervention. Biochem Soc Trans 2003; 31: 462-4.

51 Seeman E, Vellas B, Benhamou C et al. Strontium ranelate reduces the risk of vertebral and nonvertebral fractures in women eighty years of age and older. 7 Bone Miner Res 2006a; 21: 1113-20.

52 Seeman E, Vellas B, Benhamou CL et al. Sustained 5-year vertebral and non-vertebral fracture risk reduction with strontium ranelate in elderly women with osteoporosis. Osteoporos Int 2006b; 18:2

53 Roux C, Isaia G, Cannata Andia JB, Devogelaer JP. Strontium ranelate reduces the risk of vertebral fracture in young postmenopausal women with severe osteoporosis. Ann Rheum Dis 2007; 66 (Suppl 11): 681.

54 Reginster JY. Advances in the treatment of postmenopausal osteoporosis [French]. Rev Med Liege 2007; 62: 352-9.

55 Borgström F, Jönsson B, Ström O, Kanis JA. An economic evaluation of strontium ranelate in the treatment of osteoporosis in a Swedish setting: based on the results of the SOTI and TROPOS trials. Osteoporos Int 2006; 17 : 1781-93. Epub 2006 Sep 29.

56 McCombs JS, Thiebaud P, McLaughlin-Miley C, Shi J. Compliance with drug therapies for the treatment and prevention of osteoporosis. Maturitas 2004; 48: 271-87.
Correspondence:

Stephen I'Anson, Just Medical Media Ltd, 11 Redgrove Park, Cheltenham, Gloucestershire, GL51 6QY, UK. Email: publisher@justmedicalmedia.com. 